\title{
Factors associated with lumbo-pelvic pain in recreational cyclists
}

\author{
M Rodseth, 1 MSc (Physiotherapy), A Stewart, 2 PhD \\ ${ }^{1}$ Strada Aron Cotruș, Bucharest sector 1, Romania 014131 \\ 2 Department of Physiotherapy, School of Therapeutic Sciences Faculty of \\ Health Sciences University of the Witwatersrand, Johannesburg, South \\ Africa
}

Corresponding author: A Stewart (aimee.stewart@wits.ac.za)

Background: Overuse injuries in cyclists are as high as $85 \%$, with lower back and pelvis pain (LBPP) being common. The lower back and pelvis are pivotal to powering and controlling the bicycle and essential for optimal functioning, comfort and performance. Cyclists spend long, continuous hours in sustained forward flexion, which is regarded as a main contributor to LBPP. Cyclists with LBPP assume greater lumbar flexion but the reason has not yet been established.

Objectives: To identify intrinsic and bicycle set-up factors associated with lumbo-pelvic pain in cyclists.

Methods: This study was cross-sectional and descriptive. One hundred and twenty-one cyclists in Gauteng, South Africa, participated in this study. The factors proposed to be associated with LBPP were determined to be namely: lumbar curvature on the bicycle in all three handlebar positions, strength of the gluteus maximus (Gmax) and medius (Gmed), extensibility of the hamstrings, control of lumbar movement in the direction of flexion, neurodynamics, active straight leg raise, one leg stance test for lateral pelvic shift, leg length discrepancy and bicycle set-up (saddle height, set-back and angle, handlebar height, forward reach, cleat position).

Results: Only the lumbar curvature in the brake lever position $(p=0.03)$ and weakness of the Gmed $(p=0.05)$ were related to LBPP in cyclists.

Conclusion: This study was the first to assess the relationship between the multiple factors described above and LBPP in cyclists. Understanding the relationship between increased lumber flexion in the brake lever position and the weakness of the Gmed and LBPP may lead to the development of strategies to reduce LBPP occurrence.

Keywords: bicycle set-up, load transfer, low back, motor control, physical pain

\section{S Afr J Sports Med 2017;29:1-8. DOI: 10.17159/2078-516X/2017/v29i0a2406}

The prevalence of non-traumatic cycling injuries, including lumbo-pelvic pain (LBPP), is estimated to be as high as $85 \%$, with the influencing factors for the development of this type of pain in cyclists being: training, the physical aspects of cycling and bicycle set-up factors. [1,2,]. Previously investigated factors include: (1) association between training factors and LBPP, (2) kinematics and position of the lower back on the bicycle (3) surface EMG of the musculature of the hip, lumbar, thoracic areas and upper limbs. ${ }^{[1,2]}$ These studies had very small sample sizes and did not test specific postural or movement dysfunctions. None of them investigated physical and bicycle set-up factors.

The position of the cyclist on the bicycle is influenced by movement in two directions - forwards and backwards between the saddle and handlebars, and from side to side. ${ }^{[3]}$
The seated position of the cyclist leads to an increased tendency towards a "round-back" posture, emphasised by the increased forward bent position assumed to reduce aerodynamic drag. [1] Sustained end-range forward lumbar flexion during cycling could be pivotal in the development of LBPP. [2.3] Cyclists with LBPP assume greater lower lumbar flexion compared to asymptomatic cyclists ${ }^{[2]}$, supporting the hypothesis that LBPP is related to this position.

The lower back and pelvis absorb and distribute loads from the legs, providing a stable base to control and power the bicycle. [3] Integrated functioning of the muscle system is essential for optimal movement and stability of the lumbopelvic spine. ${ }^{[3]}$ Movement occurs through the pathway of least resistance, whereby more flexible structures compensate for less flexible ones creating stress and strain in a specific direction. ${ }^{[4]}$ With repetitive loading, this direction-specific hypermobility is reinforced, resulting in tissue damage, pain and uncontrolled movement. ${ }^{[4]}$

As cyclists habitually use the gluteus maximus muscle (Gmax) in an elongated position [5], resulting in "stretchweakness" of the muscle, they place an increased demand on their hamstrings to compensate for changes in the lengthtension relationship. The increased demand on the hamstrings through the combined effects of a weak, elongated Gmax and increased knee flexion moment created by using cleated pedals results in hypertrophy and increased passive stiffness of the hamstrings. [6] The imbalance in the passive stiffness of the hamstrings and lumbo-pelvic musculature induces increased movement in the lumbo-pelvic area and over time results in joint hypermobility, leading to micro- and eventual macrotrauma of the spinal structures. ${ }^{[6]}$

Weakness of the gluteus medius muscle (Gmed) in individuals with low back pain leads to increased side-toside/lateral shift of the pelvis with a subsequent loss of pelvic control. The poor endurance of this muscle can also result in early-onset pelvic rotation [7] and, combined with frequent movement in the increased range, in joint hypermobility causing micro- and eventual macro-damage of lumbo-pelvic structures. The increased lateral shift of the pelvis during the weight shifting of pedalling, combined with lumbo-pelvic musculature impairment (especially the Gmed) in transferring loads between the trunk and legs, can lead to LBPP.

Besides the position of the cyclist, the bicycle may influence the development of LBPP. Therefore proper bicycle set-up is essential for injury prevention, safety, comfort, and peak performance. ${ }^{[8]}$ With cycling, the asymmetrical variables of the body have to adapt to the symmetrical design of the bicycle to function as one unit as a result of the abnormal stress loads being placed on tendons and muscles. Optimal fitting of the bicycle to the cyclist's body geometry should result in less stress and strain, decreasing injury incidence ${ }^{[9]}$ but few studies have investigated the association between LBPP and bicycle set-up. ${ }^{[3,9]}$ Cyclists have three contact points with the bicycle (saddle, handlebars and pedals) that determine the forwardbackward and side-to-side position critical for effective transmission of force to the pedals and optimal performance of the cyclist.

The aim of this study was therefore to identify factors 


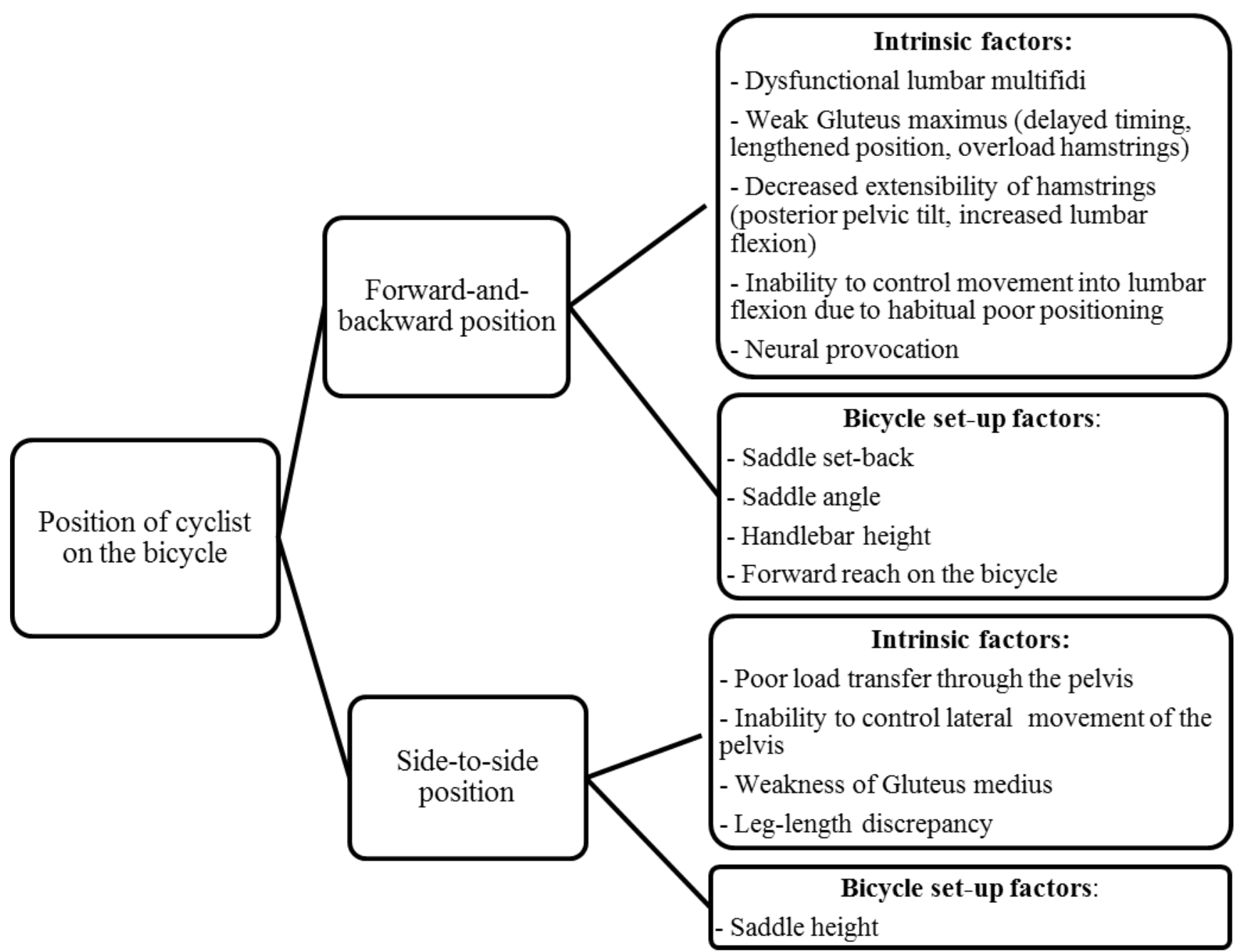

Fig. 1. Factors influencing forward-and-backward and side-to-side position on the bicycle

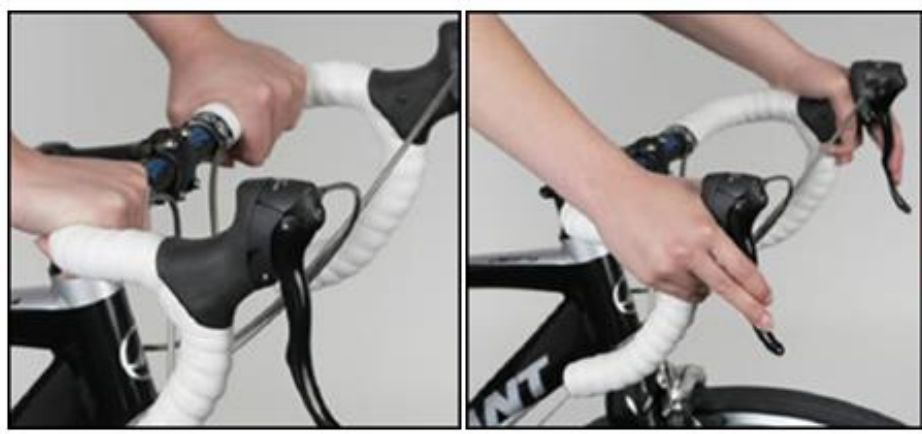

possibly associated with LBPP in cyclists in Gauteng and establish this relationship. It was hypothesised that factors that could influence the forward-backward and side-to-side position of the cyclist on the bicycle, as illustrated in Figure 1, could contribute to the development of LBPP.

\section{Methods}

\section{Participants}

This cross-sectional descriptive study included cyclists who were 18-years or older; who had cycled for more than one year; who cycle more than three, but less than 12 hours/week (in the last 2 months); had participated in at least one road race longer than $90 \mathrm{~km}$ but fewer than 20 races per year; used a racing/road bicycle during training and racing on road; used

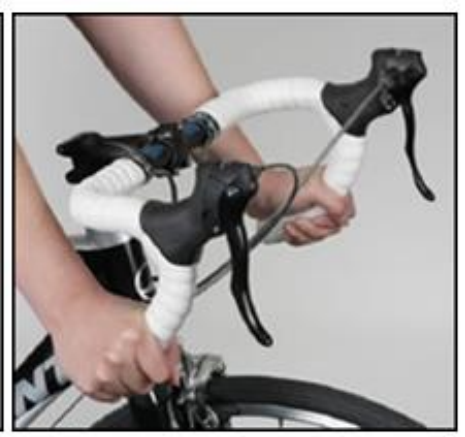

Fig. 2. Illustration of handlebar positions.

Left to right: Upright seated position, Brake lever position, Drop position

cleats; had no injuries to the spine in the preceding two years nor specific structural pathology of the spine or spinal surgery. Participants' informed consent and institutional ethical approval was obtained (Human Research Ethics Committee University of the Witwatersrand M110649).

\section{Assessments}

Assessments were undertaken by the first author of factors hypothesised to contribute to the development of LBPP, as recommended by an expert panel of physiotherapists, given the lack of literature. These included anthropometric; intrinsic physical and bicycle set-up factors. Thirteen cyclists were included in a pilot study to assess the repeatability of the measurements of the physical factors and assessed twice, one week apart. All measurements were done on a treatment plinth 
in a seated or horizontal position, with feet supported.

Body mass index (BMI) was calculated using body weight [electronic digital bathroom scale (Carmen Care) (kg)] divided by height [portable stadiometer (HS, Scales2000) (m)]. BMI has a sensitivity of $83 \%$ and specificity of $76 \%$ in detecting body fat percentage at $25.5 \mathrm{~kg} / \mathrm{m}^{2}$.

A Saunders digital inclinometer (Saunders Group Inc., Minnesota, USA) was used to measure lumbar angles/curvature in three positions: seated upright (hands on transverse bar of handlebars), brake lever (hands on brake hoods) and "drops" (hands on drops) (Figure 2). Participants performed a few pedalling cycles per riding position before stopping with pedals at the 3 o'clock and 9 o'clock positions, right foot forward. [11] The lumbar flexion curvature was calculated by subtracting the measurement at L5/S1 from T12/L1; with each of the three positions measured thrice and the means of the three measures calculated. The intra-class correlation coefficient (ICC) and reliability coefficients for the static lumbar position and lumbo-sacral angle range from 0.91 to 0.97 for intra-rater reliability respectively and $0.63-0.75$ for inter-rater reliability. ${ }^{[10]}$

The inner range holding capacity of the Gmax was assessed with the participant prone, with only trunk supported, in the neutral lumbar position; feet on floor, knees slightly flexed. ${ }^{[5]}$ Two pressure biofeedback units (PBU) (Chattanooga) were placed under the left and right anterior superior iliac spine (ASIS) and inflated to $20 \mathrm{mmHg}$. An assessment was made of the passive range of hip extension, knee in $90^{\circ}$ flexion, while the lumbo-pelvic area was stabilised in neutral. A rod was positioned to touch the posterior aspect of the thigh when the hip was in the horizontal $\left(0^{\circ}\right)$ position. The participant lifted one leg at a time into hip extension, knee in $90^{\circ}$ flexion, until the posterior thigh touched the rod, maintaining contact, as well as neutral lumbo-pelvic alignment, for 15 seconds while the lifting, holding and lowering of the leg was measured with the PBU meters. Normal inner range control of the Gmax was taken into consideration when participants had successfully completed the movement twice. Reliability studies have been done on the use of a dynamometer in determining the strength of the Gmax during prone hip extension and on the reliability for assessing lumbar movement during passive hip extension ( $\mathrm{k}=0.72-0.76$ and ICC of $0.69-0.85$ ) ${ }^{[4]}$, but no reliability or validity studies have been done on the assessment of both through range control and the inner range holding capacity of the Gmax in a prone position, with only trunk support.

Hamstring extendibility was assessed while supine, with the test leg in $90^{\circ}$ hip flexion, thigh supported, knee comfortably flexed. The knee of the test leg was passively extended until the onset of firm resistance or a strong stretch sensation was felt. ${ }^{[11]}$ The knee extension angle (KEA) was measured with a digital inclinometer and repeated three times per leg. ${ }^{[11]}$ Hamstring length is regarded as normal if the KEA for both legs is less than $20^{\circ}$ and there is excellent reliability (intra-rater ICC $=0.90-0.98$, inter-rater ICC $=0.90$ ). ${ }^{111]}$

The participant was in the side lying position, with the lumbar spine and pelvis in neutral alignment and the underneath leg slightly flexed. A combination of tests was used to measure full range control and the ability to hold the inner range capacity of the Gmed. ${ }^{66,12]}$ Full passive range of motion was assessed by lifting the top leg into hip extension, external rotation and abduction (Ext/Abd/ER) stabilising the neutral lumbo-pelvic position, and noting when the hip reached the benchmark of $45^{\circ}$ abduction (marker/rod positioned). The participant then actively lifted the top leg (Ext/Abd/ER) to the marker, maintained controlled contact for 15 seconds before smoothly lowering the leg. An inability to maintain neutral alignment of the lower back and pelvis resulted in test failure. Two smoothly controlled repetitions without substituting with the hip, lower back or pelvic movements indicated through range control of the deep posterior Gmed. Reliability studies have been done on concentric and eccentric strength of the Gmed using a dynamometer at neutral hip alignment and on pelvic control during active hip abduction. No reliability or validity studies have been done on the assessment of both full range control and inner range holding capacity of the Gmed in the side lying position. ${ }^{[12]}$

Control of lumbar flexion was measured with the sitting forward lean test, ${ }^{[13]}$ knees and hips at $90^{\circ}$ and the participant's lower back in a visually estimated neutral position. The S1 vertebra and a point $10 \mathrm{~cm}$ above this area were marked (flexible tape measure). The participant had to keep the lower back in neutral with the two points $10 \mathrm{~cm}$ apart while leaning forward to $120^{\circ}$ of hip flexion (goniometer). Five practice runs were done with verbal and tactile input to maintain the neutral lumbar curvature; then five times without feedback, measuring the distance between the two marks to the nearest millimetre and calculating the mean. Maintaining $10 \mathrm{~cm}$ between the marks, or a changed position of less than $1 \mathrm{~cm}$, was an indication of adequate flexion control of lumbar flexion. ${ }^{[14]}$ This test has excellent inter-rater reliability (ICC of $0.96, n=40$ ). ${ }^{[13]}$

Neural mobility was assessed with the slump test (reflecting the lumbar position often assumed by cyclists when riding), following a six-stage sequence. This test was considered to be positive if the participant's symptoms were reproduced at any point of the sequence and alleviated with the release of neck flexion. This test has excellent inter-rater reliability $(\mathrm{k}=0.83$; ICC $=0.70-0.92$ ) and intra-rater reliability (ICC $=0.80-0.95 ; \mathrm{r}=0.88$ ). [4]

The active straight leg raise test (ASLR) in the supine position was used to assess load transfer between the trunk and legs. It was proposed to assess force closure around the pelvis by assessing the amount of effort used for a low load activity. ${ }^{[14]}$ The participant raised a straight leg $20 \mathrm{~cm}$ off the bed successively, rating perceived effort on a six point scale (0-5) This was repeated twice and the means calculated. The scores of both sides were added, resulting in a score ranging from 0 10. It was considered positive if the mean was greater than one and negative if less than one. ${ }^{[15]}$ There is substantial inter-rater reliability ( $\mathrm{k}=0.70$ for left ASLR and $\mathrm{k}=0.71$ for right) in patients with chronic NSLBP. [14]

Lateral shift of the pelvis was measured using the single-leg stance movement control test. ${ }^{[5]}$ Participants stood in a normal upright position with feet one-third of their trochanteric distance apart and the umbilicus aligned with an upright pole. 
They then shifted their weight onto the left leg, followed by the right leg (standing on one leg). The lateral movement of the umbilicus from the midline was measured with a spirit level ruler at completion of weight transfer. This was repeated three times to each side. The means of the weight shift to the left and right sides were calculated and considered within normal limits if the shift was less than $10 \mathrm{~cm}$ for each leg and the difference in the shift between legs was less than $2 \mathrm{~cm}$. ${ }^{[4]}$ There is excellent intra-rater reliability ( $\mathrm{k}=0.84$ and $\mathrm{k}=0.67$ for left and right leg respectively) and moderate to substantial inter-rater reliability ( $\mathrm{k}=0.65$ for left and $\mathrm{k}=0.43$ for right) for this test. ${ }^{[4]}$

Leg length discrepancy (LLD) was measured in the supine position from ASIS to the most distal part of the lateral malleolus (LM) with a flexible tape measure. ASIS to LM was preferred over ASIS to medial malleolus (MM) as it limits the influence of the contour of the thigh, provides a more direct line of measurement, and also has excellent reliability (intrarater reliability $\mathrm{ICC}=0.88-0.99$, inter-rater $\mathrm{ICC}=0.83$ ). Two measurements were averaged and the measured difference in the lengths was divided into three categories: discrepancies less than $6 \mathrm{~mm}, 10 \mathrm{~mm}$ and $20 \mathrm{~mm}$ respectively.

\section{Bicycle set-up measures}

The bicycle set-up was measured with the bicycle positioned on a pre-measured bicycle stand. Saddle height was measured by assessing the knee flexion angle with the pedal at bottom dead centre (BDC) and the foot parallel to the ground and aligned forward using a goniometer. This was repeated three times per leg and considered acceptable if the knee flexion was between $25-35^{\circ}$ for both legs. [16] For saddle setback a plumb line was dropped from the posterior aspect of the patella, with the crank arm of the tested leg in the horizontal forward position ( $3 \mathrm{o}^{\prime}$ clock). Intersection of the pedal axle indicated a proper setback. [8, 9] The saddle angle was measured with a digital spirit level balanced from the midline touching the front and back of the saddle and recorded as level, anteriorly or posteriorly tilted, noting the magnitude of the inclination. A level or anteriorly tilted saddle was acceptable for optimal saddle angulation. ${ }^{[8]}$

Handlebar height was calculated by subtracting the handlebar height (floor to the top of the stem of the handlebars) from the saddle height (floor to the top centre of the saddle). A height of $5-8 \mathrm{~cm}$ below the saddle indicated a proper bicycle set-up. ${ }^{[8]}$ This means that there is excellent intra-rater reliability for the distance from the handlebars to the floor $(\mathrm{ICC}=0.98)$ and the seat to the floor $(\mathrm{ICC}=0.98)(\mathrm{n}=13)$. [10]

Reach distance is defined as the distance between the saddle and the handlebars, including arm and upper body length, which has a direct impact on the position of the lumbar spine and pelvis. ${ }^{[9]}$ Reach distance consists of the three factors involved in reaching forward from the saddle: the distance from the back of the saddle to the transverse bar of the handlebars; full arm length (acromion to metacarpal heads) and upper body length (from flat surface of plinth to incisura jugularis of the manubrium sterni in supported sitting). These were measured three times, and the means calculated and matched with the recommended reach distances. ${ }^{[9]}$

Cleat position was measured by palpating and marking the first metatarsal head while the participant was standing. The midfoot cleat position of the shoe was within limits if found to be in line with the first metatarsal head. ${ }^{[8,9]}$

The reliability and validity of the bicycle set-up measures, except for handlebar height, have not been reported. ${ }^{10]}$

\section{Statistical analysis}

From a cross-sectional study it is expected that following univariate analysis no more than 10-12 factors would be associated with low back pain when testing at the liberal 0.15 level of significance. These factors were then analysed using a logistic regression and usually 10-15 subjects need to be included for each factor. ${ }^{[17]}$ Therefore at least 120 volunteers were included. In a univariate analysis, participants with and without low back pain were compared using the two sample Student's t-test and Mann-Whitney rank sum, Pearson's Chisquare test or Fisher's exact test. Factors at $p<0.20$ were included into a multivariate analysis. From the multivariate analysis (logistic regression), odds ratios and their 95\% confidence intervals (CI) were calculated for included factors. Testing was at the 0.05 level of significance, using Stata Release 12.0 statistical software.

\section{Results}

The intra-rater reliability was excellent for the majority of the physical tests (ICC/Kappa >0.70), except for the lateral sway to the right, slump (final category), the Gmax (final category) and the Gmed (final category) $(\mathrm{p}<0.70)$. Excellent intra-rater reliability (ICC / Kappa >0.70) was obtained for the bicycle setup measures, except for the saddle height $(\mathrm{p}<0.70)$.

Of the 121 participants who volunteered to participate in the physical assessment, $80 \%(n=97)$ were males and $20 \%(n=24)$ females. The mean age per gender was 47 years $( \pm 11)$ for the males and 42 years $( \pm 8)$ for the females. Of the 121 participants, $74 \%(n=90)$ experienced LBPP during or after cycling. Seventynine percent of them $(n=71)$ were males and $21 \%(n=19)$ were females. Pain during or after cycling was mostly reported around the sacroiliac joint compared to $41 \%$ with central low back pain and $27 \%$ with unilateral low back pain. The time to the onset of LBPP during cycling was between one-two hours for $28 \%$ of cyclists. For $51 \%$ this was experienced after more than two hours of cycling mostly while in the brake lever position $(62 \%)$ or seated upright $(41 \%)$ position. However, on the whole, training was not affected by pain $(43 \%)$ or participants trained through pain $(40 \%)$. For a summary of the physical and bicycle set-up assessments, see Figures 3 and 4. In the univariate analysis, only lumbar curvature was related to LBPP ( $p=0.01-0.02$ ). From the univariate analysis, all factors with a significance value of less than 0.2 were included in a multivariate analysis (Figure 5). In the multivariate analysis, only lumbar curvature in the brake lever position $(\mathrm{p}=0.03 ; \mathrm{CI}$ : 1.00-1.09) and weakness of the Gmed ( $\mathrm{p}=0.05$; CI: 0.98-11.94) were associated with LBPP (Table 1). The risk for LBPP increased by 1.01 times for every degree of lumbar flexion added in the brake lever position. Participants with weakness of the Gmed were also 3.4 times more likely to develop LBPP 
Lumbar position on bicycle Brake levers

- Slump position/Lx flexion $(87.60 \%)$

- Mean curvature: $15.93^{\circ}$

$(\mathrm{SD}: 10.11)(\mathrm{p}=0.01)$

- LBPP group: $88.89 \%$ in Lx

flexion

Seated in upright position

$-86.78 \%$ in Lx flexion - Mean curvature: $15.23^{\circ}$ (SD: 10.31)

$(\mathrm{p}=0.01)$

-LBPP group: $87.78 \%$ in Lx

flexion

\section{Drop position}

$-92.56 \%$ in Lx flexion - Mean curvature: $17.94^{\circ}$ (SD: 9.65) $(\mathrm{p}=0.02)$

-LBPP group: $91.11 \%$ in Lx flexion

\section{Leg-length discrepancy}

$-76.86 \%$ less than $10 \mathrm{~mm}$ difference in leg-length $(\mathrm{p}=0.68)$

$-61.16 \%$ less than $6 \mathrm{~mm}$ difference $\quad(\mathrm{p}=0.68) \quad$ and $2.48 \%$ more than $20 \mathrm{~mm}$ difference $(\mathrm{p}=0.16)$

- Mean LLD: 0.632 (SD: 0.060) $(\mathrm{p}=0.67)$

-LBPP group: $77.78 \%$ less than $10 \mathrm{~mm}$

$62.22 \%$ less than $6 \mathrm{~mm}$ and

$1.11 \%$ more than $20 \mathrm{~mm}$ difference

\section{BMI $(\mathrm{p}=0.20)$}

- An almost equal number of participants presented with normal BMI $(\mathrm{n}=55,45.5 \%)$ or were overweight $(\mathrm{n}=51$, $42.2 \%)$

- Mean BMI (SD): 25.98

(3.77) $\mathrm{kg} / \mathrm{m}^{2}(\mathrm{p}=0.24)$

-LBPP group: $\quad(\mathrm{n}=44,89 \%)$ normal BMI, $(\mathrm{n}=46,51.11 \%)$ overweight/obese

- Mean BMI (SD): 25.8 (3.8) $\mathrm{kg} / \mathrm{m}^{2}$

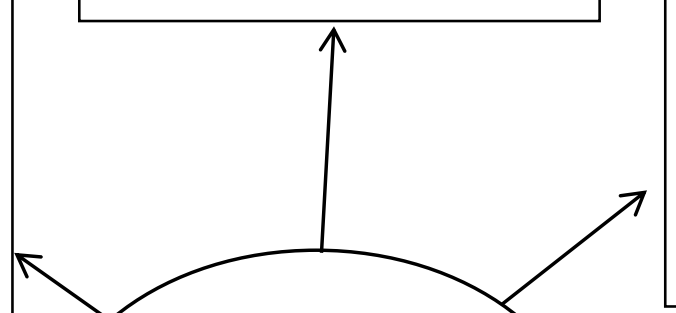

Lumbo-pelvic stability

Active straight leg raise $(\mathrm{p}=0.67)$

- $(\mathrm{n}=78,64.5 \%)$ normal ASLR

- LBPP group: $(n=59,65.6 \%)$ normal ASLR

Lateral sway $(\mathrm{p}=0.19)$

- normal lateral sway $(\mathrm{n}=74,61.2 \%)$

- Mean sway left (SD): $7.86(1.94) \mathrm{cm}$

- Mean sway right (SD): $7.21(1.92) \mathrm{cm}$

-LBPP group: normal lateral sway $(\mathrm{n}=52,57.8 \%)$

\section{Sitting forward lean $(\mathrm{p}=0.68)$}

- no lumbar flexion give with sitting

forward lean test $(n=114,94.2 \%)$

- Mean lean (SD): $0.3(0.38) \mathrm{cm}$

-LBPP group: no flexion give/normal test $(\mathrm{n}=84,93.3 \%)$

\section{Muscle tests \\ Hamstring length $(\mathrm{p}=0.81)$}

- presenting with shortened hamstrings

- KEA > $20^{\circ}(\mathrm{n}=84,69.42 \%)$

- Mean Left KEA (SD): $23.73^{\circ}$ (11.71)

$(\mathrm{p}=0.22)$

- Mean Right KEA (SD): $23.52^{\circ}$

(11.11) $(\mathrm{p}=0.80)$

- LBPP group: decreased length/KEA

$>20^{\circ}(\mathrm{n}=63,70 \%)$

Gmax inner range holding $(\mathrm{p}=1.00)$

- presenting with poor Gmax inner range holding capacity $(\mathrm{n}=99,81.8 \%)$

-LBPP group: poor control $(\mathrm{n}=73$, $81.1 \%)$

Neural mobility $(\mathrm{p}=0.23)$
$\begin{array}{llr}\text { Slump } & & \\ -70.25 \% & \text { presented } & \text { with } \\ \text { normal slump test/no } & \text { sle } & \text { neurodynamic dysfunction - -- } \\ \text {-LBPP } & \text { group: } 83.33 \% \\ \text { presented with normal slump }\end{array}$

Gmed through range control $(\mathrm{p}=0.12)$ -poor control ( $\mathrm{n}=107,88.4 \%)$

-LBPP group: insufficient control $(\mathrm{n}=82,91.1 \%)$

Fig. 3. Summary of the physical characteristics of cyclists

(Table 1). Gender was associated with BMI $(p=0.005)$, the Gmax inner range holding capacity $(p=0.006)$, hamstring length ( $p=0.001)$, the Gmed through range control $(p=0.003)$ and thoracolumbar and lumbosacral angles and curvatures $(\mathrm{p}=0.001-0.04)$ in all handlebar positions.

Distance cycled per week was associated with gender $(p=0.012)$. Statistically significant relationships were found between BMI and the Gmed ( $\mathrm{p}=0.01)$, thoracolumbar angle $(\mathrm{p}=0.001)$ and lumbar lordosis $(\mathrm{p}=0.001-0.004)$ in all positions.

Only BMI $(p=0.01)$, inner range holding capacity of the Gmax $(p=0.001)$ and hamstring length $(p=0.02)$ had a significant relationships with the Gmed. Participants with poor through range control of the Gmed also had poor Gmax inner range holding $(\mathrm{n}=92 ; 85.98 \%)$ and decreased hamstring flexibility $(n=78 ; 72.90 \%)$. Holding capacity of the Gmax $(p=0.01)$ and control of the Gmed $(p=0.021)$ were associated with the length of the hamstrings. If hamstring length was poor, insufficient inner range control of the Gmax ( $n=74,88.10 \%)$ and the Gmed control $(n=78,92.86 \%)$ also presented.

Hamstring length was associated with lumbosacral angle (L5/S1) in the seated upright ( $\mathrm{p}=0.03 ; \mathrm{CI}: 27.18-30.19)$, drops $(\mathrm{p}=0.03$; CI: $39.69-42.51)$ and brake lever positions $(\mathrm{p}=0.07$; CI: 31.71-34.60).

The Gmax inner range holding capacity was associated with 
Reach $(\mathrm{p}=0.29)$

- cyclists with an incorrect reach distance $(\mathrm{n}=110,91 \%)$

- cyclists bunched up (reach forward too short) $(\mathrm{n}=52.9$, $53 \%)$.

- LBPP group: bunched up $(\mathrm{n}=49,54 \%)$

- Reach ratio: $\mathrm{p}=0.52$

Handlebar height $(\mathrm{p}=0.49)$

- cyclists with the handlebar height out of the recommended limit of $5-8 \mathrm{~cm}$ below the saddle $(n=84,69 \%)$

- handlebars too high $(\mathrm{n}=66$, $55 \%$ )

-LBPP group: out of the recommended range $(n=64$, $71 \%$ ), with handlebars again set too high $(\mathrm{n}=53,59 \%)$

\section{Cleat position $(\mathrm{p}=0.55)$}

- cyclists - cleats positioned incorrectly on shoes $(\mathrm{n}=68$, $56 \%$ )

- LBPP group: cleats positioned incorrectly on shoes $(\mathrm{n}=52,58 \%)$

\section{$\sqrt{1}$}

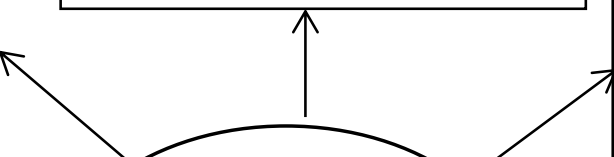

\section{Position of the saddle}

Saddle height $(\mathrm{p}=0.19)$

- cyclists' saddle height out of the recommended range $(\mathrm{n}=78,65 \%)$

- Saddle too high $(\mathrm{n}=13,11 \%)$

- Saddle too low $(n=2622 \%)$

- Asymmetry between left and right sides in $34 \%(n=41)$

- LBPP group: saddle height out of range $(n=55,61 \%)$, presenting with asymmetry between sides $(\mathrm{n}=26$, $29 \%)$ or too low saddle $(\mathrm{n}=20$, $22 \%)$

\section{Saddle set-back $(\mathrm{p}=0.25)$}

- presenting with a saddle set-back out of the recommended range $(n=73,60 \%)$ of which the saddle was set too far forward - LBPP group saddle set-back not in recommended range $(n=57,63 \%)$.

\section{Saddle angle $(\mathrm{p}=0.21)$}

- saddles tilted anteriorly $(\mathrm{n}=58$, $48 \%)$ followed by $(n=47,39 \%)$ tilted posteriorly $(\mathrm{p}=0.51)$

- Mean (SD) tilt: $0.72^{\circ} \quad$ (2.5) $(\mathrm{p}=0.44)$

- LBPP group: saddles tilted anteriorly $(\mathrm{n}=45,50 \%)$ followed by $(\mathrm{n}=32,36 \%)$ tilted posteriorly

- Mean (SD) tilt: $0.81^{\circ}$

$(\mathrm{p}=0.44)$

Fig. 4. Summary of the bicycle set-up factors

lateral sway $(\mathrm{p}=0.031)$, Gmed control $(\mathrm{p}=0.001)$ and hamstring length $(\mathrm{p}=0.007)$.

Saddle angle was associated with thoracolumbar angle $(\mathrm{T} 12 / \mathrm{L} 1)$ in the handlebar drops position ( $\mathrm{p}=0.02$; CI: 57.7560.30).

Lumbar curvature in the handlebar drops position was associated with the sitting forward lean test ( $p=0.04$; CI: 16.20 19.68), while the lumbosacral angle and curvature were consistently related to sitting forward lean test.

\section{Discussion}

Flexion of the lumbar spine in the brake lever position and weakness of the Gmed were associated with LBPP, with most cyclists experiencing pain in the brake lever position. This was also the most frequently adopted position in training (48\% of time was spent in this position in cyclists with and without LBPP). Cyclists assume a position of lumbar flexion on the bicycle, regardless of the level of competition, and those with LBPP adopt greater lumbar flexion, ${ }^{[1,2]}$ as was also seen here.
The mechanism by which increased lumbar flexion leads to LBPP is, however, not clear. [2,] The authors assessed several factors that could influence this position (including an inability to prevent/control lumbar flexion), but none were associated with LBPP. None of the factors, besides gender $(p=0.03)$ and BMI ( $p=0.002$ ), were related to the lumbar curvature in the brake lever position. Other studies suggest the flexion relaxation phenomenon or mechanical creep but with inconclusive outcomes. ${ }^{[2,10]}$ Poor position sense (proprioception) with subsequent spinal repositioning error could also contribute to the increased lumbar flexion.

Lack of through range control of the Gmed was associated with LBPP. Most of the participants (88\%) were unable to concentrically shorten their Gmed to inner range, isometrically hold inner range, and eccentrically control the return, keeping a neutral alignment of the lumbar spine and pelvis. In this study, $91 \%$ of those with LBPP were unable to do so. Neumann ${ }^{[18]}$ reported an increase in hip internal rotation at greater ranges of knee flexion. Cyclists are positioned in hip flexion and use increasing ranges of flexion and internal rotation during 


\section{Factors taken to multivariate analysis $(\mathbf{p}<0.2)$}

- Distance cycled per week $(\mathrm{p}=0.19)$

- Lateral sway $(\mathrm{p}=0.19, \mathrm{n}=38,42.2 \%)$

- $\mathrm{LLD}>20 \mathrm{~mm}(\mathrm{p}=0.16, \mathrm{n}=1,1.1 \%)$

- Gmed control $(\mathrm{p}=0.12, \mathrm{n}=82,91.1 \%)$

- Lx curvature in the brake lever position $(\mathrm{p}=0.01)$

- $\mathrm{Lx}$ curvature in the seated upright position $(\mathrm{p}=0.01)$

- Lx curvature in the drops position $(\mathrm{p}=0.02)$

- Saddle height $(\mathrm{p}=0.19, \mathrm{n}=55,61.1 \%)$

- Handlebar height $(\mathrm{p}=0.15)$ leading to overuse, hypertrophy and extensibility loss. The influence of the Gmed in the development of LBPP needs to be interpreted with caution and the reliability for assessing control of the Gmed improved.

Eighty-one percent of participants had an elongated Gmax, (poor inner range holding). ${ }^{[5]}$ Most cyclists use their Gmax in a lengthened position only, needing inner range contraction and increased strength when they stand up out of the saddle, resulting in greater hip extension. Muscle fatigue ${ }^{[6]}$, which was not investigated, may be the reason why none of the other factors were associated with LBPP.

Although bicycle set-up is often regarded as the cause of LBPP ${ }^{[8]}$, no bicycle factors were related to LBPP in this study. The assessment of a static set-up compared to a dynamic set-up should be considered, as the movement and position of the lumbo-pelvic spine changes substantially during cycling.

\section{Conclusion}

The results of this study suggest that flexion of the lumbar spine in the brake lever position and weakness of the Gmed are associated with LBPP.

\section{References}

1. Burnett AF, Cornelius MW, Dankaerts $\mathrm{W}$, et al. Spinal kinematics and trunk muscle activity in cyclists: a comparison between healthy controls and non-specific chronic low back pain subjects - a pilot investigation. Man Ther 2004; 9:211-219. doi: 10.1016/j.math.2004.06.002

Fig. 5. Summary of factors taken to the multivariate analysis

pedalling. ${ }^{[21]}$ Habitual use of increased hip internal rotation and hip adduction will lead to weakness of the Gmed, resulting in more hip adduction and lateral shift which could induce an increase in lumbo-pelvic rotation. This could in turn lead to micro- and macro-trauma of the lumbo-pelvic structures. [6]

The Gmed is responsible for $70 \%$ of the mediolateral stability of the pelvis; weakness thereof could result in poor lateral control, presenting as an increase in lateral pelvic shift as mechanical loads are transferred from the legs through the pelvis with pedalling. ${ }^{[19]}$ Lateral pelvic tilt (side-to-side rocking) occurs naturally during cycling, while exaggerated at high speeds and increased fatigue. [3] With poor lateral control, side-to-side translation is exaggerated inducing a side flexion and/or rotation moment through the lower back and pelvis ${ }^{[1]}$ resulting in increased mobility and microdamage of lumbosacral structures. ${ }^{\left[{ }^{6}\right]}$ Sustained flexion with rotation is implicated in the injury of passive spinal structures, such as intervertebral discs, with resultant micro-damage to the annulus fibrosis. An unexpected finding was that there was no relationship between the one leg stance test and Gmed strength $(\mathrm{p}=0.24)$, considering its primary role of stabilising the pelvis during the one leg stance. ${ }^{[1]}$ This might be as a result of the Gmed primarily control pelvic tilt as opposed to pelvic shift when other muscles such as the Gmax are activated. ${ }^{[14]}$

Weakness in the Gmed was related to Gmax weakness $(p=0.001)$ and decreased the extensibility of the hamstrings $(p=0.02)$ but neither were related to LBPP. This relationship might be explained by a global muscle system dysfunction, where weakness in the global stabilisers (Gmed and Gmax) increases the load on the global mobilisers (hamstrings)
2. Van Hoof W, Volkaerts K, O'Sullivan K, et al. Comparing lower lumbar kinematics in cyclists with low back pain (flexion pattern) versus asymptomatic controls - field study using a wireless posture monitoring system. Man Ther 2012; 17:312317. doi: 10.1016/j.math.2012.02.012

3. Mellion MB. Neck and back pain in bicycling. Clin Sports Med 1994; 13:137-164. PMID: 8111848

4. Luomajoki H, Kool J, de Bruin ED, et al. Reliability of movement control tests in the lumbar spine. BMC Musculoskelet Disord. 2007;8:90.doi: 10.1186/1471-2474-90

5. Richardson CA, Sims K. An inner range holding contraction: An objective measure of stabilising function of an antigravity muscle. In: Proceedings of the World Confederation for Physical Therapy: $11^{\text {th }}$ International Congress London, UK: 1991: 829-831.

6. Sahrmann S. The hip as a factor in low back pain: Evidence why relative flexibility is key. J Orth Sports Phys Ther; 2012; 42:A1114. doi: 10.2519 /jospt.2012.0302

7. Lee SP, Powers C. Description of a weight-bearing method to assess hip abductor and external rotator muscle performance. J Orthop Sports Phys Ther 2013; 43:392-397. doi: 2519/jospt .2013.4412

8. Silberman MR, Webner D, Collina S,et al. Road bicycle fit. Clin J Sport Med 2005; 15:271-276. doi: 10.1097/01.jsm.0000 171255.70156.da

9. de Vey Mestdagh K. Personal perspective: in search of an optimum cycling posture. Appl Ergon 1998; 29:325-334. PMID: 9703347

10. Schulz SJ, Gordon SJ. Riding position and lumbar spine angle in recreational cyclists: A pilot study. Int J Exerc Sci 2010; 3: 174181. PMCID: PMC4738870

11. Davis DS, Quinn RO, Whiteman CT, et al. Concurrent validity of four clinical tests used to measure hamstring flexibility. J Strength Cond Res 2008; 22:583-588. doi: 


\subsection{9/JSC.0b013e31816359f2}

12. Comerford M, Mottram SL, Gibbons SGT. Understanding movement and function concepts. Kinetic control Movement Dysfunction Course. London 2004. www.rehabtrainer.com. $\mathrm{au} /$ courses/resources/essentials/core-stability.pdf

13. Enoch F, Kjaer $\mathrm{P}$, Elkjaer $\mathrm{A}$, et al. Inter-examiner reproducibility of tests for lumbar motor control. BMC Musculoskel Disord 2011; 12:114. doi: 10.1186/1471-2474-12114

14. Roussel NA, Nijs J, Truijen S, et al. Low back pain: clinimetric properties of the Trendelenburg test, active straight leg raise test, and breathing pattern during active straight leg raising. J Manipulative Physiol Ther 2007; 30:270-278. doi: 10.1016/j.jmpt.2007.03.001
15. Mens JM, Huis In't Veld YH, et al. The Active Straight Leg Raise test in lumbopelvic pain during pregnancy. Man Ther 2012; 17:364-368. doi: 10.1016/j.math.2012.01.007

16. Peveler W, Bishop P, Smith J, Richardson M, Whitehorn E. Comparing methods for setting saddle height in trained cyclists. J Exerc Physiol Online. 2005; 8:51-55.

17. Nunnally JC. Psychometric theory. $2^{\text {nd }}$ ed.. New York: McGraw-Hill, 1978.

18. Neumann DA. Kinesiology of the hip: a focus on muscular actions. J Orthop Sports Phys Ther 2010; 40:82-94. doi: 10.2519/jospt.2010.3025

19. Grimaldi A. Assessing lateral stability of the hip and pelvis. Man Ther 2011; 16: 26-32. doi: 10.1016/j.math.2010.08.005 